\title{
ЭКОЛОГИЧЕСКИЙ МОНИТОРИНГ ПОРОДНЫХ ОТВАЛОВ ГОРНОПРОМЫШЛЕННЫХ АГЛОМЕРАЦИЙ
}

\author{
Высоцкий Сергей Павлович 1 , \\ sp.vysotsky@gmail.com \\ Козырь Дмитрий Александрович2, \\ kozyr.dmitriy@gmail.com \\ 1 Донбасская национальная академия строительства и архитектуры, \\ Украина, 286123, г. Макеевка, ул. Державина, 2. \\ 2 Донецкий национальный технический университет, \\ Украина, 283001, г. Донецк, ул. Артема, 58.
}

\begin{abstract}
Актуальность. На данный момент на территории Донбасса находится около 600 породных отвалов. Их горение приводит к выбросам сероводорода, диоксида серы и других парниковых газов в атмосферу. Важной экологической задачей является предотвращение образования эндогенных очагов на отвалах угольных шахт и уменьшение загрязнения атмосферы. Цель: совершенствование мониторинга уровня экологической опасности отвалов угольных шахт, находящихся в горнопромьшленных агломерациях для своевременной локализации и предотвращения образования очагов горения на породных отвалах. Объект: породные отвалы угольных шахт.

Методы: применение теории подобия и анализа размерностей между породным отвалом и атмоссрерой с учетом дополнения Хантли; натурные и лабораторные исследования влияния условий и расстояния тепловизионной съемки, скорости ветра на фиксируемую энергетическую светимость; исследования выбросов токсичных газов при горении породного отвала.

Результаты. Моделирование основных теплофизических характеристик между очагом тепловыделения на породном отвале угольной шахты и тепловизором позволило описать их уравнением критериального вида. Экспериментальными исследованиями установлено, что при увеличении расстояния тепловизионной съемки снижается фиксируемая энергетическая светимость. Зависимость энергетической силы света от ракурса тепловизионной съемки в интервале углов 0-60 $\mathrm{coответ-}$ ствует закону Ламберта. Установлено, что температурный контраст между тепловизором и очагом тепловыделения обратно пропорционален дистанции тепловизионной съемки в степени 1,81. Экспериментально обоснован процесс образования сероводорода на породных отвалах. Установлено, что повышение концентрации одного из самых токсичных компонентов выбросов горящего породного отвала - сероводорода, находится в степенной зависимости от относительной влажности атмосферного воздуха. Обоснована эфффективность экологического мониторинга породных отвалов с применением дистанционных способов контроля - тепловизоров и дистанционно-пилотируемых летательных аппаратов. Применение дистанционного тепловизионного мониторинга уровня экологической опасности породных отвалов с использованием дистанционнопилотируемых летательных аппаратов и достоверного определения выбросов в атмосфрерный воздух при горении породных отвалов снизит стоимость работ по сравнению с действующей контактной методикой в 5 раз.
\end{abstract}

\section{Ключевые слова:}

Экологический мониторинг, породный отвал, очаг самовозгорания, тепловизор, контроль теплового состояния, дистанционные методы.

\section{Введение}

С опасностью возгорания породных отвалов (ПО) сталкиваются многие горнодобывающие страны мира (Россия, Китай, Германия, США) [1]. После окончания эксплуатации в ПО процессы горения могут продолжаться более 10 лет [2]. Проблема горящих ПО актуальна и для Донбасса. Так, например, в Луганской Народной Республике заскладировано более 1500 млн т отходов промышленности, что составляет 110 тыс. т на км² территории [3].

Поскольку ПО расположены в промышленных агломерациях, в ряде случаев их санитарно-защитная зона (С33) не выдержана и содержит жилую застройку [4]. Выбросы при горении ПО содержат $\mathrm{NO}_{2}, \mathrm{SO}_{2}$ и $\mathrm{H}_{2} \mathrm{~S}, \mathrm{CO}, \mathrm{CO}_{2}, \mathrm{NH}_{3}, \mathrm{HCl}$, моноароматические углеводороды, бензол, тяжелые металлы ( $\mathrm{Hg}, \mathrm{As}, \mathrm{Pb}, \mathrm{Se})$ [5]. Исследования выбросов при горении ПО в Польше показали, что концентрации $\mathrm{CH}_{4}$ и $\mathrm{CO}_{2}$ в среднем составляют 42,7 и 7160 ppm соответственно. Эти уровни являются вредными для здоровья и показывают, что горение ПО может быть опасным и в течение нескольких лет после окончания его эксплуатации. В очагах горения концентрации достигают 5640 ppm $\mathrm{CH}_{4}$ и 51976 ppm $\mathrm{CO}_{2}$ [6]. Анализ газовых компонентов выбросов в атмосферу от горящих ПО в Австралии показал, что выбросы диоксида углерода составляют от 12 до 8200 кг/м² поверхности отвала в год [7]. Известно, что горение одной тонны породных отвалов генерирует 99,7 кг СO; 0,61 кг $\mathrm{H}_{2} \mathrm{~S} ; 0,03$ кг $\mathrm{NO}_{x}$; 0,84 кг $\mathrm{SO}_{2}$ и 0,45 кг пыли [8].

При определенных условиях могут произойти взрывы горящих ПО. В 1966 г. в г. Димитров произошёл взрыв горящего ПО, в результате которого часть горящей породы попала на населенный пункт. Такие же явления произошли на нескольких угольных шахтах в Китайской Народной Республике, в регионах с длительными проливными дождями [9]. На текущий момент в ДНР горящими являются 140 ПО. В Ростовской области в состоянии горения находится более 40 отвалов [10]. 
Процесс самовозгорания ПО зависит от внутренних и внешних факторов, таких как петрологический состав, влажность и сернистость породы, температура окружающей среды и т. д. Кроме этого, при расположении ПО в зоне с высокой проницаемостью, связанной с участками пониженного рельефа, могут возникнуть благоприятные условия для попадания газов в тело ПО и его дальнейшего самовозгорания [11].

Одним из таких горящих ПО Донбасса является породный отвал шахты «Октябрьский рудник». Расчет рассеивания выбросов горящего ПО шахты «Октябрьский рудник», выполненный в программе ЭОЛ2000, показал превышение нормативов по сероводороду на границе расположения С33 (3 максимальных разовых предельно допустимых концентраций (ПДК)) и на местонахождении селитебной территории (рис. 1). Также на рис. 1 приведены изолинии максимальных концентраций сероводорода в долях ПДК.

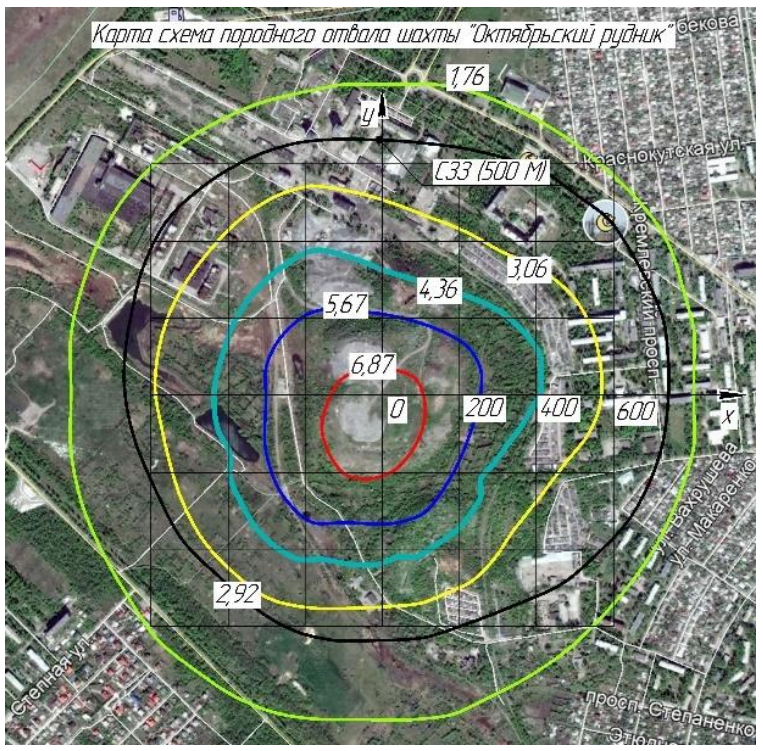

Рис. 1. Результаты расчёта рассеивания сероводорода при горении породного отвала иахтьл «Октябрьский рудник» $(2,92 ; 3,06 ; 4,36 ; 5,67 ; 6,87$ максимальные концентрации сероводорода в долях ПДК)

Fig. 1. Results of hydrogen sulfide dispersion during combustion of the dump of the Oktyabrsky mine (2,92; 3,06; 4,36; 5,67; 6,87 - maximum concentration of hydrogen sulfide in fractions of maximum permissible concentrations)

Методы и технологии борьбы с эндогенными пожарами на ПО делятся на категории: охлаждение ПО инертными газами; технологии тушения с антипирогенными добавками; технология земляных работ (обшивка, разборка и др.); технология экранирования с помощью абсорбционных траншей и контролируемое дожигание [12].

Применение своевременного мониторинга теплового состояния ПО позволит локализовать очаги самовозгорания и применить необходимые мероприятия по ликвидации очагов на ранней стадии горения. Проведение мониторинга уровня экологической безопасности ПО позволит снизить выбросы вредных веществ в атмосферу и повысит уровень экологической безопасности горнопромышленных агломераций.

Мониторинг уровня экологической безопасности ПО может проводиться дистанционным и контактным способом. Тепловизионная техника и самолеты для целей экологического мониторинга ПО впервые были использованы в 60-е гг. ХХ в. в США [13]. Тепловизионная техника также применялась для оценки теплового состояния ПО в Китае и Индии $[14,15]$. Исследователями на горящих ПО Верхнесилезского угольного бассейна в Польше были проведены натурные исследования, состоящие из серии измерений с применением тепловизионной камеры и дистанционно-пилотируемых летательных аппаратов (ДПЛА), а также контактных измерений. Использование только одного типа данных (точечные контактные измерения или тепловизионные изображения) сильно ограничивает возможности достоверного распознавания очагов самовозгорания [16].

При использовании спутниковых данных исследователями был предложен индекс интенсивности самовозгорания (SHII - self-heating intensity index), ocнованный на максимальном и минимальном значениях, взятых из спутниковых тепловых карт горящих отвалов угольных шахт. Индекс позволяет классифицировать пожары на ПО по уровням тепловой интенсивности, а именно экстремальный $(>7)$, продвинутый (3-7), средний $(3-1,5)$, начальный $(1,5-1)$, без активности $(<1)[17]$.

Исследования теплового состояния отвалов с применением спутникового мониторинга выявили основные недостатки этого метода - снижение периодичности мониторинга из-за наличия облачности, эффективность при измерении температуры только больших пожаров и низкая разрешительная способность теплового сканирования [18, 19].

В настоящее время контроль уровня экологической опасности ПО в Донецкой Народной Республике и Российской Федерации проводится контактным способом с применением термопар. Недостатки этого метода (невозможность провести измерения температуры в очаге горения, не фиксируются очаги размером до 10 м и т. д.) приводят к определению выбросов загрязняющих веществ по грубым данным [20].

Используемые в настоящее время дистанционные методы теплового контроля ПО не учитывают влияние условий и расстояния съёмки, теплопроводимых свойств породы и атмосферы. Таким образом действующие методы дистанционного контроля не позволяют достоверно выявлять температурное состояние ПО [21].

Целью работы является повышение экологической безопасности породных отвалов за счёт их мониторинга с применением дистанционных методов.

\section{Материалы и методы}

В результате проведённых исследований основных параметров взаимодействия между очагом тепловыделения на поверхности ПО и атмосферой с применением теории подобия и анализа размерностей с учётом дополнения Хантли получено уравнение крите- 
риального вида, характеризующее теплообмен между поверхностью ПО и атмосферой [22]:

$$
\begin{gathered}
\mathrm{Nu}_{\mathrm{a}}=N u_{\text {п }}^{0,5}\left(\frac{\lambda_{\mathrm{\Pi}} d}{\lambda_{\mathrm{a}} L}\right)^{0,83}, \\
\mathrm{Nu}_{\mathrm{a}}=\frac{\alpha_{\mathrm{a}} L}{\lambda_{\mathrm{a}}}, \\
\mathrm{Nu}_{\text {п }}=\frac{\alpha_{\text {п }} d}{\lambda_{\text {п }}} .
\end{gathered}
$$

где $\mathrm{Nu}_{\mathrm{a}}$ - критерий Нуссельта, характеризующий количество теплоты от очага на поверхности ПО в атмосферу; $\mathrm{Nu}_{п}-$ критерий Нуссельта, характеризующий тепловой поток к породе; $\alpha_{\mathrm{a}}-$ фиксируемый коэффициент теплоотдачи в атмосферу, Дж/( $\left.\mathrm{M}^{2} \cdot \mathrm{K} \cdot \mathrm{c}\right)$; $\alpha_{\Pi}-$ коэффициент теплоотдачи к породе, Дж/( $\left.\mathrm{M}^{2} \cdot \mathrm{K} \cdot \mathrm{c}\right)$; $L$ - дистанция между очагом тепловыделения и тепловизором, м; $\lambda_{\mathrm{a}}, \lambda_{\text {п }}-$ коэффициент теплопроводности атмосферы и породы соответственно, Дж/( $\left.\mathrm{M}^{2} \cdot \mathrm{K} \cdot \mathrm{c}\right) ; d-$ эквивалентный диаметр очага тепловыделения, м.

Критерий Нуссельта, характеризующий количество теплоты от очага на поверхности ПО в атмосферу, прямо пропорционален квадратному корню из критерия Нуссельта, который характеризует тепловой поток к поверхности ПО и зависит от теплопроводных свойств породы и атмосферы [22].

Температурный контраст $(\Delta T)$ между температурой, фиксируемой тепловизором $\left(T_{\mathrm{a}}, \mathrm{K}\right)$, и температурой очага самовозгорания $\left(T_{\Pi}, \mathrm{K}\right)$ будет равен:

$$
\begin{gathered}
\alpha_{\mathrm{a}}=\sigma\left(T_{\mathrm{n}}^{4}-T_{\mathrm{a}}^{4}\right), \\
\Delta T=T_{\text {п }}-T_{\mathrm{a}},
\end{gathered}
$$

где $\sigma$ - постоянная Стефана-Больцмана (Дж·c $\left.{ }^{-1} \cdot \mathrm{M}^{-2} \cdot \mathrm{K}^{-4}\right)$.

$$
\Delta T=\frac{\left(\frac{\alpha_{\mathrm{n}} d}{\lambda_{\text {п }}}\right)^{0,5}\left(\frac{\lambda_{\mathrm{n}} d}{\lambda_{\mathrm{a}} L}\right)^{0,83} \lambda_{\mathrm{a}}}{T_{\mathrm{n}}^{3}\left(1+\left(\frac{T_{\mathrm{a}}}{T_{\text {п }}}\right)^{3}+\left(\frac{T_{\mathrm{a}}}{T_{\mathrm{n}}}\right)^{2}+\frac{T_{\mathrm{a}}}{T_{\text {п }}}\right) L \sigma} .
$$

Влияние расстояния съемки, ракурса и скорости потоков воздуха на фиксируемую энергетическую светимость очага тепловыделения было экспериментально изучено в натурных и лабораторных условиях.

При экспериментальных исследованиях в помещении устанавливали нагревательный прибор, к которому прикреплялся лист из стали и экраны разной формы и одной площади $\left(0,5 \mathrm{~m}^{2}\right)$. При исследованиях измеряли температуру и влажность воздуха в помещении, температуру нагревательного прибора контактным способом и тепловизором. При лабораторных исследованиях изменялась дистанция съемки (3-27 м), её ракурс $\left(30^{\circ}, 60^{\circ}, 90^{\circ}\right)$ и скорость воздуха (0,5-2,8 м/с). Измерения проводились с применением тепловизора Land Cyclops Ti-814 (рис. 2) [23].

Натурные исследования проводились с использованием тепловизионной камеры Fluke Ti-125 на ПО пяти шахт Донецкого региона: им. С.М. Кирова, «Октябрьский рудник», им. М.И. Калинина, им. В.И. Ленина и 13-бис (рис. 3).

При проведении исследований измерялась относительная влажность атмосферного воздуха и его температура, проводилось измерение скорости потоков воздуха, учитывалось альбедо поверхности ПО.
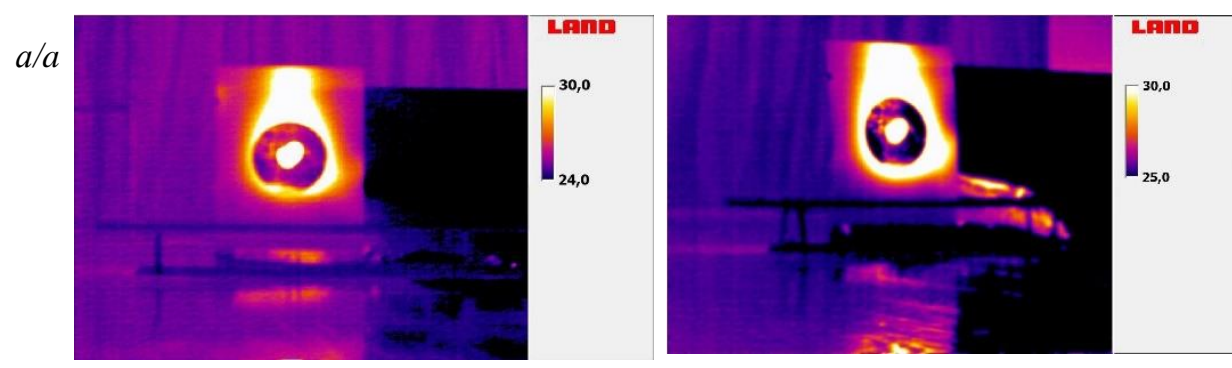

$\sigma / b$
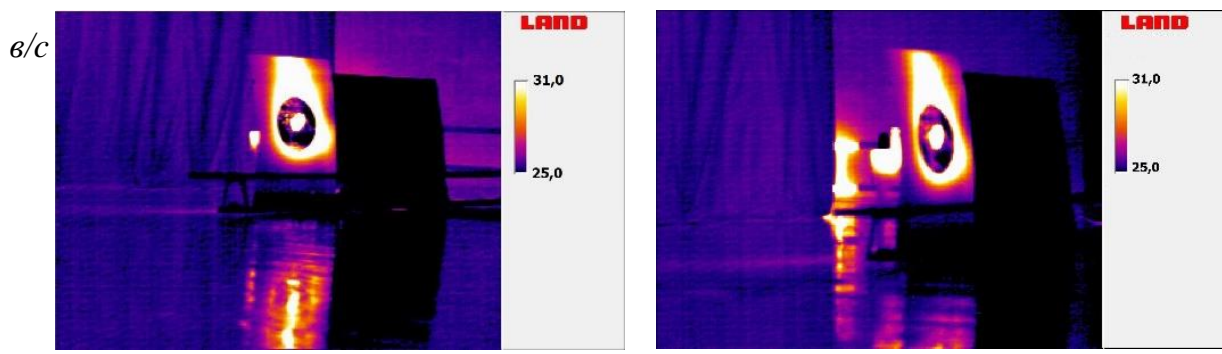

$2 / d$

Рис. 2. Тепловизионные фотографии объекта тепловыделения при расстоянии 9 м и ракурсах съёмки $90^{\circ}\left(\right.$ a), $60^{\circ}(б)$, $45^{\circ}(8), 30^{\circ}(2)$

Fig. 2. Thermal photographs of the heat release object at a distance of $9 \mathrm{~m}$ and shooting angles $90^{\circ}(\mathrm{a}), 60^{\circ}(\mathrm{b}), 45^{\circ}(\mathrm{c}), 30^{\circ}(\mathrm{d})$

Натурные исследования ПО проводились с четырех сторон с учетом ракурса съемки. На выявленных очагах тепловыделения выполнялась дополнительная съемка с изменением расстояния и ракурса. Также на очагах тепловыделения температура измерялась контактным спосо- бом. При лабораторных и натурных исследованиях было сделано более 220 измерений энергетической светимости (рис. 3). Используемые в исследованиях средства измерения были метрологически обеспечены. Результаты исследований были статистически обработаны [23]. 

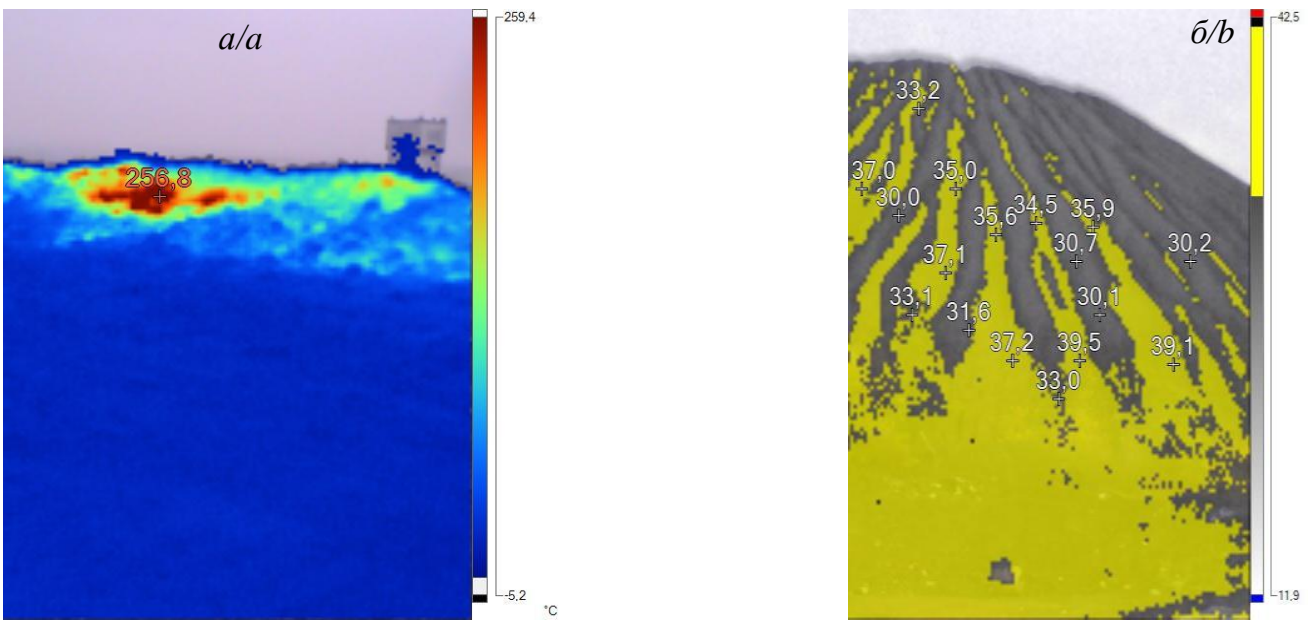

Рис. 3. Тепловизионные фотографии натурных исследований (а - ПО шахты «Октябрьский рудник»; б - ПО шахты 13-я бис)

Fig. 3. Thermal imaging photographs of field studies ( $a$ - the waste dump of the «Oktyabrsky mine»; $b$ - the waste dump of the $13^{\text {th }}$ bis mine)

Натурные исследования породных отвалов показали, что в местах ПО, подверженных эрозии, повышение температуры составило от 0,9 до $6,1{ }^{\circ} \mathrm{C}$ относительно поверхности отвала. Установлено, что площадь участков поверхности породных отвалов с повышенной температурой составляет 20-25\% (рис. 3, б).

\section{Результаты исследования}

Проведенные экспериментальные исследования установили, что увеличение расстояния дистанционной съемки приводит к снижению фиксируемой энер- гетической светимости очага тепловыделения по степенной зависимости (рис. 4).

Лабораторные исследования показали, что увеличение скорости потоков воздуха приводит к снижению фиксируемой энергетической светимости. Так, к уменьшению фиксируемой энергетической светимости на 29 \% привело увеличение скорости воздуха до 2,8 м/с. Изменение фиксируемой энергетической светимости под влиянием скорости потоков воздуха согласуется с теоретическими основами.

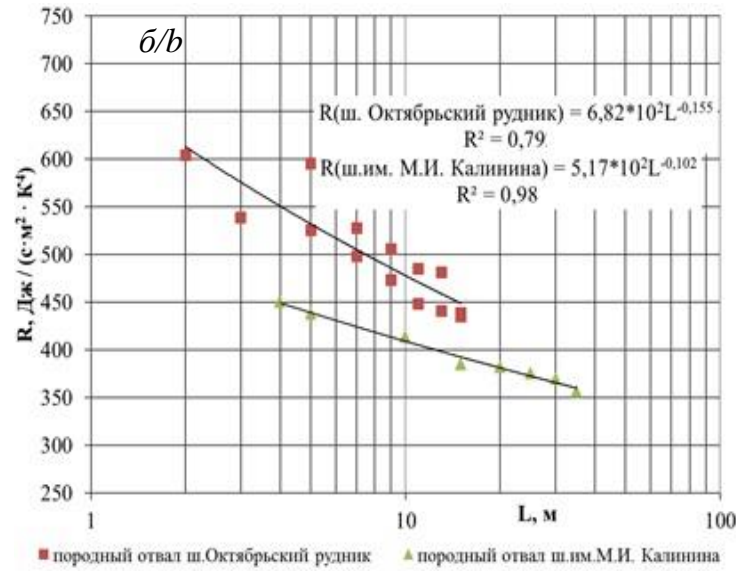

Pис. 4. Изменение фиксируемой энергетической светимости $(R)$ в зависимости от расстояния съемки (L) при ракурсе $90^{\circ}$ при лабораторных (а) и при натурных исследованиях (б)

Fig. 4. Change in fixed radiant luminosity $(R)$ versus shooting distance $(L)$ at an angle of $90^{\circ}$ in laboratory (a) and in field studies $(b)$

Проведенные исследования показали, что зависимость энергетической силы света от ракурса тепловизионной съемки в интервале углов $0-60^{\circ}$ полностью соответствует закону Ламберта. Изменение ракурса тепловизионной съёмки породных отвалов от $0^{\circ}$ до $60^{\circ}$ снижает фиксируемую энергетическую светимость очага тепловыделения до $50 \%$.

Сравнение фиксируемой энергетической светимости, полученной при использовании критериального уравне- ния и при натурных исследованиях, показало их соответствие. Таким образом уравнение критериального вида адекватно (рис. 5) [22]. Установлено, что величина $\Delta \mathrm{T}$ между тепловизором и очагом самонагревания при экспериментальных исследованиях обратно пропорциональна дистанции съемки (L) в степени 1,81 (рис. 6).

Экспериментальные исследования на горящем ПО шахты им. М.И. Калинина позволили провести анализ выбросов загрязняющих веществ (рис. 7). 
Исследования проводились с применением трубы, которая снижала воздействие разбавления выбросов при измерении концентраций токсичных газов. Измерения концентраций кислорода, оксидов азота, диоксида серы, оксида углерода, сероводорода и температуры газа проводились с использованием газоанализаторов ОКСИ-5M, TESTO-350XL, УГ-2 и измерителя скорости ИС-2. Анализ газовых выбросов был проведен на 26 очагах тепловыделения на поверхности породного отвала.

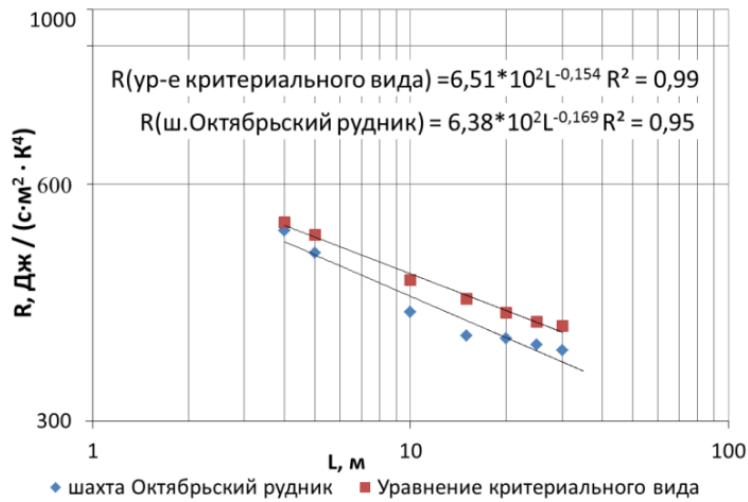

Pис. 5. Влияние расстояния съемки (L) на фиксируемую энергетическую светимость (R) при лабораторных исследованиях и при использовании уравнения критериального вида

Fig. 5. Influence of the shooting distance $(L)$ on the fixed radiant luminosity $(R)$ in laboratory studies and when using the criterial equation

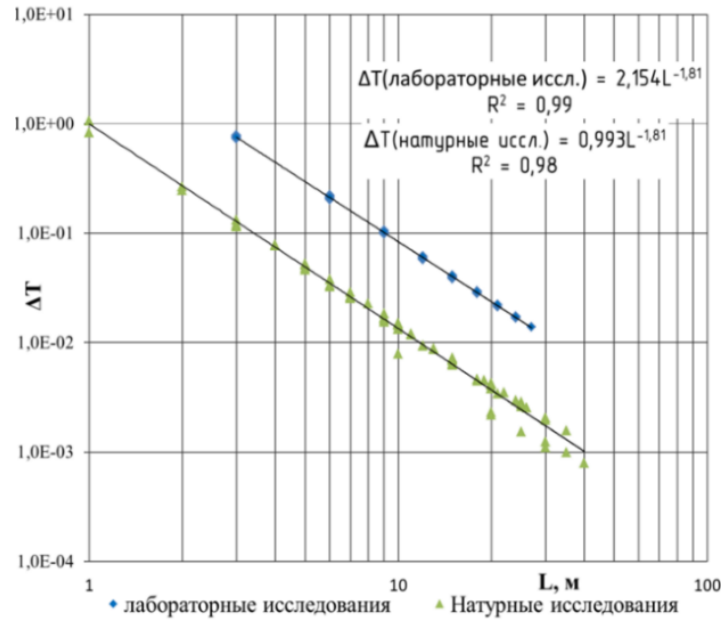

Puc. 6. Зависимость температурного контраста между очагом самонагревания и тепловизором $(\Delta T)$ от расстояния съемки $(L)$

Fig. 6. Dependence of the temperature contrast between the self-heating hotbed and the thermal imager $(\Delta T)$ on the shooting distance $(L)$

Активизация окислительных процессов в участках эрозии на поверхности ПО приводит к образованию вредных газообразных веществ. Площадь поверхности ПО, подверженная эрозии, составляет от 20 до $25 \%$ площади отвала. Повышение температуры в местах эрозии составляет от 1 до $6{ }^{\circ} \mathrm{C}$. Концентрация токсичных веществ в местах эрозии составила от 4 до
8 ПДК диоксида серы $\left(\mathrm{SO}_{2}\right)$, от 2 до 10 ПДК диоксида азота $\left(\mathrm{NO}_{2}\right)$, от 10 до 15 ПДК оксида углерода (CO).

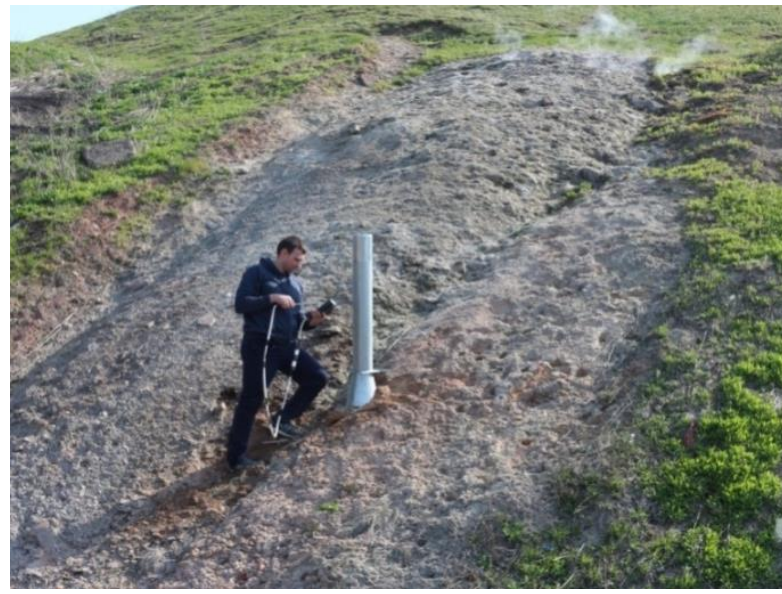

Pис. 7. Натурные исследования на отвале шахты имени М.И. Калинина

Fig. 7. Field research on the waste dump of the M.I. Kalinin mine

Для оценки влияния измеренных выбросов горящего ПО шахты им. М.И. Калинина на атмосферный воздух г. Донецка был проведен расчет рассеивания приземных концентраций в программном комплексе ЭОЛ-2000 (рис. 8).

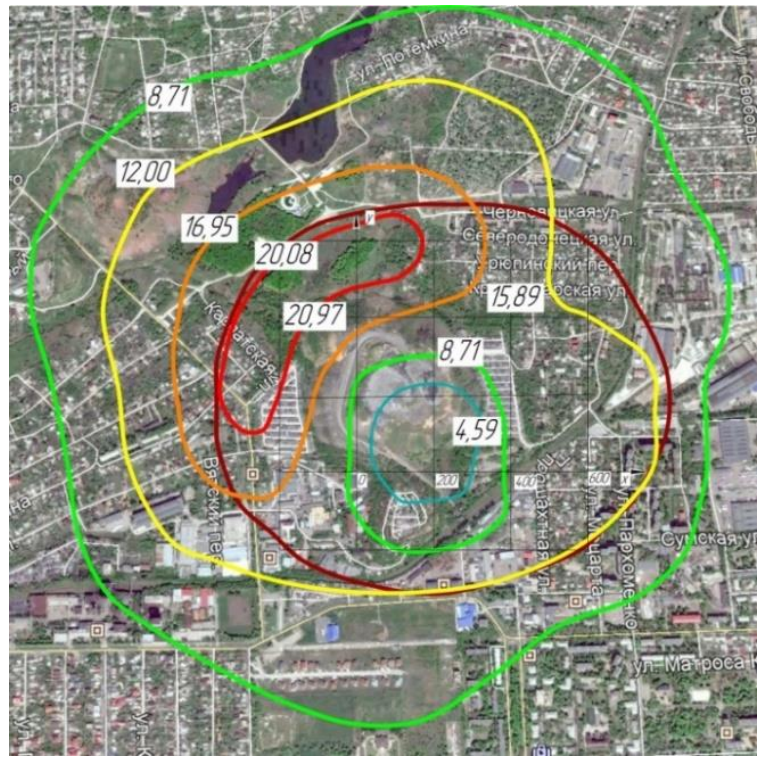

Puc. 8. Результаты расчёта рассеивания сероводорода при горении отвала шахты имени М.И. Калинина $(4,59 ; 8,71 ; 12,00 ; 15,89 ; 16,95 ; 20,08 ; 20,97$ максимальные кониентрации сероводорода в долях ПДК)

Fig. 8. Results of calculating hydrogen sulfide dispersion during combustion of the waste dump of M.I. Kalinin mine $(4,59 ; 8,71 ; 12,00 ; 15,89 ; 16,95 ; 20,08$; 20,97 - maximum concentration of hydrogen sulfide in fractions of maximum permissible concentrations)

Результаты расчета рассеивания выбросов вредных веществ показали превышение санитарных нормативов на границе С33 и селитебной территории, 
которая находится внутри С33, по следующим веществам: $\mathrm{SO}_{2}, \mathrm{NO}_{2}, \mathrm{H}_{2} \mathrm{~S}$, группа суммации $30\left(\mathrm{SO}_{2}+\mathrm{H}_{2} \mathrm{~S}\right)$, группа суммации $31\left(\mathrm{NO}_{2}+\mathrm{SO}_{2}\right)$. Максимальные концентрации зафиксированы по $\mathrm{H}_{2} \mathrm{~S}$ и по совместному действию $\mathrm{SO}_{2}$ и $\mathrm{H}_{2} \mathrm{~S}$ (группа суммации 30 ) - более 20 ПДК на С33, около 16 ПДК на селитебной территории.

Установлено, что до 50 \% выбросов загрязняющих веществ от горящего ПО составляют выбросы от зон, подверженных эрозии.

Известно, что интенсивность выбросов вредных веществ при горении ПО увеличивается при атмосферных осадках. Образование выбросов при горении ПО представляет собой совокупность химических реакций. Окисление породы происходит при окислении её минералов (пирит, гален) в присутствии кислорода и воды. В результате окисления образуются сульфатионы. Снижение кислотности среды приводит к дальнейшему окислению ионов железа до трехвалентного состояния и дальнейшему образованию гидроксида железа по следующим реакциям [24]:

$$
\begin{gathered}
\mathrm{FeS}_{2}+3,5 \mathrm{O}_{2}+\mathrm{H}_{2} \mathrm{O}=\mathrm{Fe}^{2+}+2 \mathrm{SO}_{4}{ }^{2-}+2 \mathrm{H}^{+}, \\
\mathrm{Fe}^{2+}+0,25 \mathrm{O}_{2}+\mathrm{H}^{+}=\mathrm{Fe}^{3+}+0,5 \mathrm{H}_{2} \mathrm{O}, \\
\mathrm{Fe}^{3+}+3 \mathrm{H}_{2} \mathrm{O}=\mathrm{Fe}(\mathrm{OH})_{3}+3 \mathrm{H}^{+} .
\end{gathered}
$$

Процесс образования выбросов загрязняющих веществ происходит в присутствии воды. Газовые выбросы, содержащие серу, выделяются при достижении температуры около $300^{\circ} \mathrm{C}$ в очаге горения на ПО
[25]. При этом пары воды взаимодействуют с нагретым пиритом ПО [26]:

$$
6 \mathrm{FeS}_{2}+10 \mathrm{H}_{2} \mathrm{O}=10 \mathrm{H}_{2} \mathrm{~S}+6 \mathrm{FeO}+2 \mathrm{SO}_{2} \text {. }
$$

На низкотемпературных очагах тепловыделения процесс образования сероводорода недостаточно изучен.

Учитывая, что плотность диоксида серы почти в два раза выше плотности воздуха, часть его накапливается у поверхности ПО. Сернистый ангидрид реагирует с влагой атмосферы с образованием сернистой кислоты, которая в свою очередь реагирует с сульфидом в составе ПО, и приводит к образованию сероводорода.

Для обоснования процесса образования сероводорода в низкотемпературных очагах тепловыделения проведены экспериментальные исследования по оценке влияния относительной влажности атмосферного воздуха на изменение концентрации выбросов сероводорода.

Экспериментальные исследования проводились на горящем ПО шахты им. М.И. Калинина. Измерения концентрации сероводорода $(\mathrm{C}(\mathrm{H} 2 \mathrm{~S}), \mathrm{ppm})$ и концентрации сернистого ангидрида $\left(\mathrm{C}\left(\mathrm{SO}_{2}\right), \mathrm{ppm}\right)$ выполнялись с применением газоанализатора Testo 350XL. Относительная влажность $(h, \%)$ увеличивалась при использовании пульверизатора. Измерение относительной влажности воздуха проводилось термогигро-

\begin{tabular}{|c|c|c|c|c|c|c|c|c|c|c|c|c|c|c|c|c|}
\hline$h, \%$ & 33,0 & 35,1 & 36,0 & 35,0 & 45,1 & 44,1 & 43,0 & 58,1 & 57,0 & 59,1 & 56,0 & 57,0 & 67,0 & 68,0 & 69,0 & 68,0 \\
\hline $\mathrm{C}\left(\mathrm{H}_{2} \mathrm{~S}\right), \mathrm{ppm}$ & 6,0 & 5,0 & 7,0 & 6,0 & 9,0 & 10,0 & 8,1 & 11,0 & 11,0 & 10,0 & 11,0 & 10,0 & 22,0 & 21,0 & 21,0 & 20,0 \\
\hline
\end{tabular}
метром testo 610 (рис. 9, таблица)

Таблица. Результать экспериментальньх исследований Table. $\quad$ Results of experimental studies

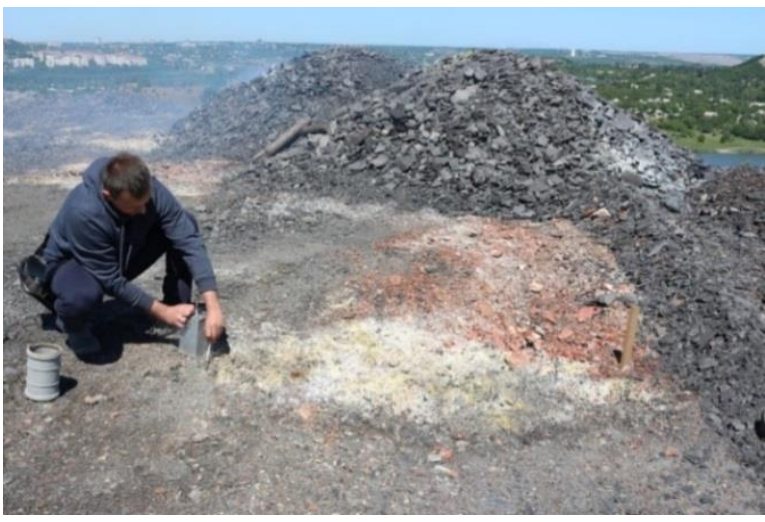

Рис. 9. Проведение измерений на отвале иахты имени М.И. Калинина

Fig. 9. Measurements at the waste dump of the M.I. Kalinin mine

В ходе экспериментальных исследований установлено, что повышение концентрации сероводорода в выбросах горящего ПО происходит при увеличении относительной влажности атмосферного воздуха.

Изменение концентрации сероводорода при изменении относительной влажности атмосферного воздуха описывается зависимостью (рис. 10):

$$
\mathrm{C}\left(\mathrm{H}_{2} \mathrm{~S}\right)=1,55 \times 10^{-2} h^{1.67}\left(\mathrm{R}^{2}=0,87\right) .
$$

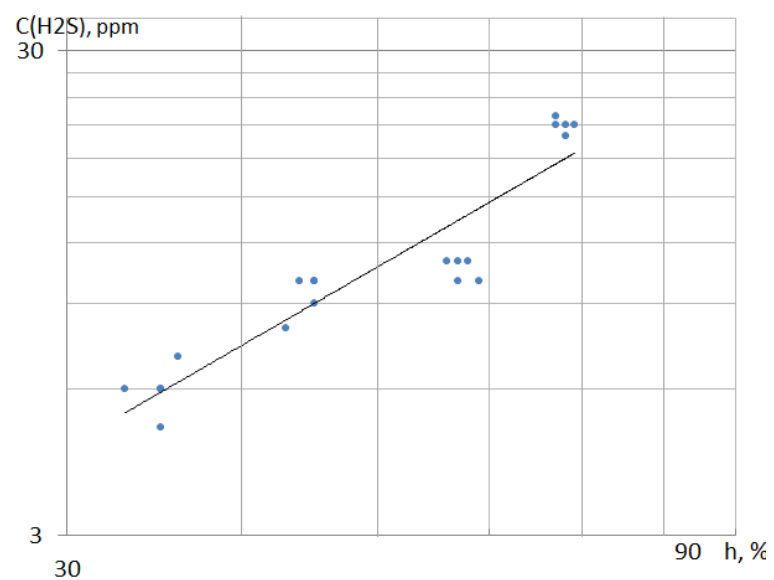

Рис. 10. Результаты экспериментальных исследований выбросов сероводорода $\mathrm{C}\left(\mathrm{H}_{2} \mathrm{~S}\right)$ и относительной влажности воздуха ( $h, \%)$

Fig. 10. Results of experimental studies of hydrogen sulfide emissions $\mathrm{C}\left(\mathrm{H}_{2} \mathrm{~S}\right)$ and relative air humidity $(h, \%)$

Взаимодействие пирита породы с сернистой кислотой, а также жизнедеятельность сульфатредуцирующих бактерий приводит к образованию сероводорода, причем этот процесс требует немало времени [27].

При проведении экспериментальных исследований установлено, что повышение влажности породы 
методом распыления воды приводит к быстрому увеличению концентрации сероводорода. Таким образом реакция пирита с сернистой кислотой является предпочтительным процессом, приводящим к повышению концентрации сероводорода.

Взаимодействие сернистого ангидрида и сероводорода проходит по процессу Клауса (рис. 11):

$$
\mathrm{SO}_{2}+2 \mathrm{H}_{2} \mathrm{~S}=3 \mathrm{~S}+2 \mathrm{H}_{2} \mathrm{O} \text {. }
$$

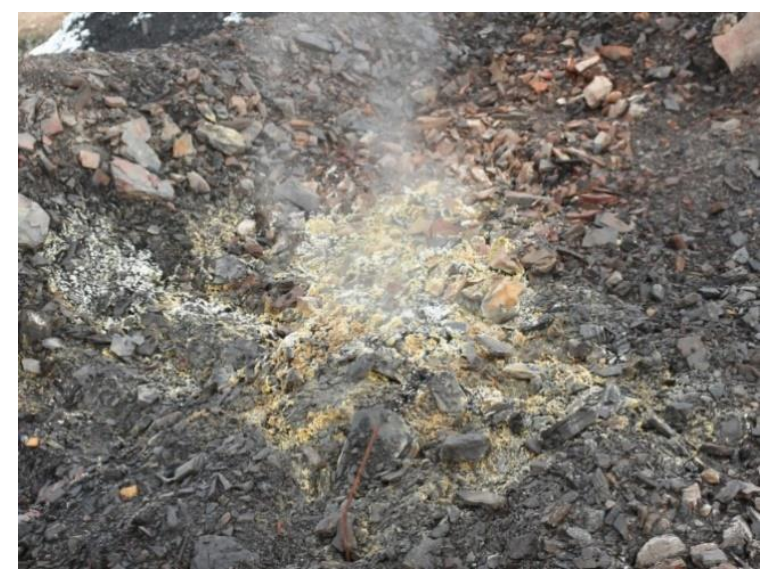

Pис. 11. Элементарная сера на поверхности отвала иахты имени М.И. Калинина

Fig. 11. Elemental sulfur on the surface of the waste dump of the M.I. Kalinin mine

Первичными мероприятиями при тушении поверхностных очагов на горящих ПО являются мероприятия по нейтрализации одного из самых токсичных компонентов выбросов - сероводорода.

Ущерб, вызванный негативным воздействием горящих ПО на здоровье населения и окружающую природную среду, а также стоимость мероприятий по тушению ПО, значительно выше стоимости мониторинга их теплового состояния.

C учетом времени достижения породой температуры самовозгорания и обоснования экономического подхода к периодичности дистанционного контроля, мониторинг уровня экологической безопасности ПО рекомендуется проводить не менее 1 раза в месяц [22]

В настоящий момент установлено горение 5 ПО в Макеевке и 29 ПО в Донецке. Для 20 \% ПО г. Донецка и для почти 50 \% всех ПО г. Макеевка не определен уровень их экологической опасности. Отсутствие мониторинга уровня экологической опасности ПО может привести не только к загрязнению атмосферы городов, но и к пожарам отвалов, обвалам их горящей массы.

Применение ДПЛА и тепловизоров позволит в течение года провести своевременный и безопасный

\section{СПИСОК ЛИТЕРАТУРЫ}

1. Sinha A., Singh V.K. Spontaneous coal seam fires: a global phenomenon // International research for sustainable control and management. Spontaneous Coal Seam Fires: Mitigating a Global Disaster. International conference. UNESCO Office Beijing. Beijing, 2005. - P. 42-66.

2. Саранчук В.И. Борьба с горением породных отвалов. - Киев: Наукова думка, 1978. -162 с. контроль теплового состояния всех горящих ПО Донецкой Народной Республики.

Применение тепловизионного мониторинга экологической опасности ПО с использованием ДПЛА снизит стоимость работ по сравнению с действующей контактной методикой в 5 раз.

\section{Заключение}

1. В ходе проведения исследований доказано, что применение дистанционного мониторинга теплового состояния отвалов угольных шахт позволит эффективно повысить уровень экологической безопасности горнопромышленных агломераций.

2. Влияние теплофизических характеристик атмосферы и породы на очагах самовозгорания отвалов описано уравнением в критериальном виде. Натурные исследования подтвердили адекватность критериального уравнения.

3. Установлено, что величина критерия Нуссельта, характеризующая тепловой поток от очага тепловыделения в атмосферу, прямо пропорциональна квадратному корню из критерия Нуссельта, который характеризует тепловой поток к поверхности породного отвала и зависит от теплопроводных свойств породы и атмосферы.

4. Теоретически обосновано и подтверждено экспериментальными исследованиями влияние ракурса и расстояния тепловизионной съёмки очагов самовозгорания на температурный контраст и фиксируемую дистанционными методами контроля энергетическую светимость. Температурный контраст между тепловизором и очагом тепловыделения, характеризующий влияние теплофизических характеристик породы и атмосферы, обратно пропорционален дистанции тепловизионной съёмки в степени 1,81. Экспериментально установлено, что изменение ракурса тепловизионной съёмки породных отвалов от $0^{\circ}$ до $60^{\circ}$ снижает фиксируемую энергетическую светимость очага тепловыделения до 50 \%. Снижение энергетической светимости соответствует закону Ламберта. Тепловизионную съёмку необходимо проводить с минимально допустимого средствами дистанционного контроля расстояния в условиях повышенной облачности при скорости ветра не более 5-7 м/с.

5. Экспериментально установлено, что до $50 \%$ выбросов загрязняющих веществ от горящего породного отвала составляют выбросы от зон терриконов, подверженных эрозии.

6. Установлено, что увеличение относительной влажности атмосферного воздуха $(h, \%)$ приводит к повышению концентрации сероводорода $\left(\mathrm{C}\left(\mathrm{H}_{2} \mathrm{~S}\right)\right)$.

3. Дрозд Г.Я. Стратегия и потенциал развития сектора обращения с отходами на примере Луганщины // Вода и экология. 2017. - № 2. - С. 60-78

4. Терриконы: монография / Л.Г. Зубова, А.Р. Зубов, А.А. Зубов, А.В. Харламова, С.Г. Воробьев, Ю.И. Макаришина, В.В. Буняченко. - Луганск: Изд-во «Ноулидж», 2015. - 712 с.

5. Influence of soil cover on reducing the environmental impact of spontaneous coal combustion in coal-waste gobs: a review and 
new experimental data / X. Querol, X. Zhuang, O. Font, M. Izquierdo, A. Alastuey, I. Castro, B.L. van Drooge, T. Moreno, J.O. Grimalt, J. Elvira, M. Cabañas, R. Bartroli, J.C. Hower, C. Ayora, F. Plana, A. López-Soler // International Journal of Coal Geology. - 2011. - V. 85. - P. 2-22.

6. Environmental influence of gaseous emissions from self-heating coal waste dumps in Silesia, Poland / M. Fabiańska, J. Ciesielczuk, Á. Nádudvari, M. Misz-Kennan, A. Kowalski, Ł. Kruszewski // Environ Geochem Health. - 2019. - V. 41 (2). - P. 575-601.

7. Greenhouse gases emissions from low-temperature oxidation and spontaneous combustion at open-cut coal mines in Australia / J.N. Carras, S.J. Day, A. Saghafi, D.J. Williams // International Journal of Coal Geology. - 2009. - V. 78 - P. 161-168.

8. Management of spontaneous combustion in coal mine waste tips in China / Ch. Liu, S. Li, Q. Qiao, J. Wang, Z. Pan // Water, Air, and Soil pollution. - 1998. - V. 103. - P. 441-444.

9. Rong-kun P., Ming-gao Y., Lai-xiang L. Experimental study on explosive mechanism of spontaneous combustion gangue dump // Journal of Coal Science and Engineering (China). - 2009. V. 15. - P. 394-398.

10. Versilov S., Vil'bitskaya N., Kurdashov V. Increase of efficiency of extinguishing of rock dumps on the surface of coal mines // IOP Conference Series: Earth and Environmental Science. - 2019. V. 272. - 022236. DOI: $10.1088 / 1755-1315 / 272 / 2 / 022236$

11. Modelling of aerodynamic process for coal waste dump located in geodynamically dangerous zone / A. Kobylkin, V. Musina, A. Batugin, V. Ponomarev, O. Vorobyeva, E. Vishnevskaya // IOP Conference Series: Earth and Environmental Science. - 2019. V. 221. - 012287. DOI: $10.1088 / 1755-1315 / 221 / 1 / 012087$

12. The mechanisms of endogenous fires occurring in extractive waste dumping facilities / K. Gogola, T. Rogala, M. Magdziarczyk, A. Smoliński // Sustainability. - 2020. - V. 12. - 2856. DOI $10.3390 / \mathrm{su} 12072856$

13. Manual of coal fire detection and monitoring, report of the project: development and implementation of a coal fire monitoring and fighting system in China / A. Rosema, H. Guan, J.L.V. Genderen, H. Veld, Z. Vekerdy, A.M.T. Katen, A. Prakash, M. Sharif. Utrecht: Netherlands Institute of Applied Geoscience, 1999. $245 \mathrm{p}$.

14. Environmental characterization of burnt coal gangue banks at Yangquan, Shanxi Province, China / X. Querol, M. Izquierdo, E. Monfort, E. Alvarez, O. Font, T. Moreno, A. Alastuey, X. Zhuang, W. Lud, Y. Wang // International Journal of Coal Geology. - 2008. - V. 75. - P. 93-104.

15. Wasilewski S., Skotniczny P. Mining waste dumps - modern monitoring of thermal and gas activities // Gospodarka Surowcami Mineralnymi - Mineral Resources Management. - 2015. V. 31 (1). - P. 155-182.

16. Abramowicz A., Chybiorz R. Fire detection based on a series of thermal images and point measurements: the case study of coalwaste dumps // The International Archives of the Photogrammetry, Remote Sensing and Spatial Information Sciences. - 2019. V. XLII. - P. 9-12.
17. Classification of fires in coal waste dumps based on Landsat, Aster thermal bands and thermal camera in Polish and Ukrainian mining regions / Á. Nádudvari, A. Abramowicz, M. Fabiańska, M. MiszKennan, J. Ciesielczuk // International Journal of Coal Science \& Technology. - 2020. URL: https://doi.org/10.1007/s40789-02000375-4 (дата обращения 26.12.2020).

18. Infrared thermography monitoring and early warning of the spontaneous combustion of coal gangue pile / Y. Wang, Y. Sheng, G. Qiang, S. Yue-yue, W. Xiu-jun, Z. Zhi-jie // The International Archives of the Photogrammetry, Remote Sensing and Spatial Information Sciences. - Beijing: XXIst International Society for Photogrammetry and Remote Sensing Congress, 2008. V. XXXVII. Part B8. - P. 203-206.

19. Uncontrolled coal fires and their environmental impacts: investigating two arid mining regions in north-central China / C. Kuenzer, J. Zhang, A. Tetzlaff van Dijk, P. Voigt, S. Mehl, H.W. Wagner // Applied Geography. - 2007. - V. 27. - P. 42-62.

20. Козырь Д.А., Костенко В. К. Обоснование методики теплового неразрушающего контроля поверхности источников горения и самонагревания на породных отвалах угольных шахт // Проблемы недропользования: Сборник научных трудов. СПб: Санкт-Петербургский горный университет, 2016. Ч. 2. - C. 104-106.

21. Высоцкий С.П., Козырь Д.А. Мониторинг теплового состояния породных отвалов с использованием дистанционных методов контроля // Вестник Академии гражданской защиты: научный журнал. - 2018. - Вып. 1 (13). - С. 59-69.

22. Высоцкий С.П., Козырь Д.А. Контроль экологического состояния породных отвалов // Вестник Донбасской национальной академии строительства и архитектуры. - 2018. - Вып. 2018 V. $3(131) .-$ C. $12-18$

23. Козырь Д.А. Усовершенствование методов контроля температуры при обеспечении экологической безопасности породных отвалов угольных предприятий // Проблемы природопользования и экологическая ситуация в Европейской России и на сопредельных территориях. - Белгород: БелГУ, 2017. C. $339-342$.

24. Высоцкий С.П., Козырь Д.А. Исследование процессов генерации выбросов токсичных газов на породных отвалах // Вестник Академии гражданской защиты: научный журнал. 2018. - Вып. 3 (15). - С. 68-74.

25. Зборщик М.П., Осокин В.В. Горение пород угольных месторождений и их тушение. - Донецк: ДонГТУ, 2000. - 180 с.

26. Физико-химические свойства углей и угольных массивов / Е.И. Захаров, Н.М. Качурин, И.И. Мохначук // Известия ТулГУ. Науки о Земле. - 2012. - № 2. - С. 58-67.

27. Lazarevic D.A. In-situ removal of hydrogen sulphide from landfill gas arising from the interaction between municipal solid waste and sulphide mine environments within bioreactor conditions Stockholm: Royal Institute of Technology, 2007. - 87 p.

Поступила 22.09.2021 г.

\section{Информация об авторах}

Высоцкий C.П., доктор технических наук, профессор кафедры техносферной безопасности Донбасской национальной академии строительства и архитектуры.

Козырь Д.А., кандидат технических наук, доцент кафедры природоохранной деятельности Донецкого национального технического университета. 
UDC 504.064

\title{
ENVIRONMENTAL MONITORING OF WASTE DUMPS OF MINING AGGLOMERATION
}

\author{
Sergey P. Vysotsky', \\ sp.vysotsky@gmail.com \\ Dmitriy A. Kozyr², \\ kozyr.dmitriy@gmail.com \\ 1 Donbas National Academy of Civil Engineering and Architecture, \\ 2, Derzhavin street, Makeevka, 286123, Ukraine. \\ 2 Donetsk National Technical University, \\ 106, B. Khmelnitsky avenue, Donetsk, 28300, Ukraine.
}

The relevance of the research. Currently, there are about 600 waste dumps in the territory of Donbass. Their combustion leads to emissions of hydrogen sulfide, sulfur dioxide and other greenhouse gases into the atmosphere. An important environmental challenge is to prevent the formation of endogenous hotbed of spontaneous combustion on the dumps of coal mines and reduce air pollution.

Purpose: to improve monitoring of the level of environmental hazard of coal mine dumps located in mining agglomerations for timely localization and prevention of the formation of hotbed of spontaneous combustion on waste dumps.

Object: waste dumps of coal mines.

Methods: application of the similarity theory and analysis of dimensions between the waste dump and the atmosphere, considering the Huntley add-on; full-scale and laboratory studies of the influence of conditions and distance of thermal imaging shooting, wind speed on the fixed radiant luminosity; studies of toxic gas emissions during combustion of waste dumps.

Results. Modeling the main thermophysical characteristics between the hotbed of spontaneous combustion on the waste dump of a coal mine and a thermal imager made it possible to describe them by a criterial equation. Experimental studies have established that with the increase in the distance of thermal imaging, the recorded energy luminosity decreases. The dependence of the energy intensity of light on the angle of thermal imaging shooting in the range of angles $0-60^{\circ}$ corresponds to Lambert's law. It was found that the temperature contrast between the thermal imager and the hotbed of spontaneous combustion is inversely proportional to the distance of thermal imaging shooting to the power of 1,81. It was established that the increase in the concentration of one of the most toxic components of emissions from a burning waste dump, hydrogen sulfide, is in power-law dependence on the relative humidity of the atmospheric air. The effectiveness of monitoring the ecological safety of waste dumps with the use of remote-control methods - thermal imagers and remotely piloted aerial vehicles - was substantiated. The use of remote thermal imaging monitoring of the level of environmental hazard of waste dumps using remotely piloted aircraft and reliable determination of emissions into the air during the combustion of waste dumps will reduce the cost of work in comparison with the current contact method by 5 times.

\section{Key words:}

Environmental monitoring, waste dump, hotbed of spontaneous combustion, thermal imager, thermal state control, remote sensing methods.

\section{REFERENCES}

1. Sinha A., Singh V.K. Spontaneous coal seam fires: a global phenomenon. International research for sustainable control and management. Spontaneous Coal Seam Fires: Mitigating a Global Disaster. International conference. UNESCO Office Beijing, 2005. pp. 42-66.

2. Saranchuk V.I. Borba s goreniem porodnykh otvalov [Combating waste dumps combustion]. Kiev, Naukova Dumka Publ., 1978. $162 \mathrm{p}$.

3. Drozd G.Ya. Strategy and potential of the waste management sector on the example of Luhansk region. Water and ecology, 2017 vol. 2, pp. 60-78. In Rus.

4. Zubova L.G., Zubov A.R., Zubov A.A., Kharlamov A.V., Vorobiev S.G., Makarishina Yu.I., Bunyachenko V.V. Terrikony: monografiya [Waste dumps: monograph]. Lugansk, Knowledge Publ. House, 2015. $712 \mathrm{p}$

5. Querol X., Zhuang X., Font O., Izquierdo M., Alastuey A., Castro I., Van Drooge B.L., Moreno T., Grimalt J., Elvira B., José J., Cabañas M., Bartroli R., Hower J., Ayora C., Plana F., LópezSoler A. Influence of soil cover on reducing the environmental impact of spontaneous coal combustion in coal-waste gobs: a review and new experimental data. International Journal of Coal Geology, 2011, vol. 85, pp. 2-22.

6. Fabiańska M., Ciesielczuk J., Nádudvari Á., Misz-Kennan M., Kowalski A., Kruszewski Ł. Environmental influence of gaseous emissions from self-heating coal waste dumps in Silesia, Poland. Environ Geochem Health, 2019, vol. 41 (2), pp. 575-601.
7. Carras J.N., Day S.J., Saghafi A., Williams D.J. Greenhouse gases emissions from low-temperature oxidation and spontaneous combustion at open-cut coal mines in Australia. International Journal of Coal Geology, 2009, vol. 78, pp. 161-168.

8. Liu Ch., Li S., Qiao Q., Wang J., Pan Z. Management of spontaneous combustion in coal mine waste tips in China. Water, Air, and Soil pollution, 1998, vol. 103, pp. 441-444.

9. Rong-kun P., Ming-gao Y., Lai-Xiang L. Experimental study on explosive mechanism of spontaneous combustion gangue dump. Journal of Coal Science and Engineering (China), 2009, vol. 15, pp. 394-398.

10. Versilov S., Vil'bitskaya N., Kurdashov V. Increase of efficiency of extinguishing of rock dumps on the surface of coal mines. IOP Conference Series: Earth and Environmental Science, 2019, vol. 272, no. 022236. DOI: 10.1088/1755-1315/272/2/022236

11. Kobylkin A., Musina V., Batugin A., Ponomarev V., Vorobyeva O., Vishnevskaya E. Modelling of aerodynamic process for coal waste dump located in geodynamically dangerous zone. IOP Conference Series: Earth and Environmental Science, 2019, vol. 221, no. 012087 . DOI: $10.1088 / 1755-1315 / 221 / 1 / 012087$

12. Gogola K., Rogala T., Magdziarczyk M., Smoliński A. The mechanisms of endogenous fires occurring in extractive waste dumping facilities. Sustainability, 2020, vol. 12, no. 2856. DOI: 10.3390/su12072856

13. Rosema A., Guan H., Veld H., Vekerdy Z., Ten Katen A.M., Prakash A. Manual of coal fire detection and monitoring. Report of the Project: Development and Implementation of a Coal Fire 
Monitoring and Fighting System in China. Utrecht, Netherlands Institute of Applied Geoscience, 1999. 245 p.

14. Querol X., Izquierdo M., Monfort E., Alvarez E., Font O., Moreno T., Alastuey A., Zhuang X., Lud W., Wang Y. Environmental characterization of burnt coal gangue banks at Yangquan, Shanxi Province, China. International Journal of Coal Geology, 2008, vol. 75. pp. 93-104.

15. Wasilewski S., Skotniczny P. Mining waste dumps - modern monitoring of thermal and gas activities. Gospodarka Surowcami Mineralnymi - Mineral Resources Management, 2015, vol. 31 (1), pp. $155-182$.

16. Abramowicz A., Chybiorz R. Fire detection based on a series of thermal images and point measurements: the case study of coalwaste dumps, The International Archives of the Photogrammetry, Remote Sensing and Spatial Information Sciences, 2019, XLII, pp. 9-12.

17. Nádudvari Á., Abramowicz A., Fabiańska M., Misz-Kennan M., Ciesielczuk J. Classification of fires in coal waste dumps based on Landsat, Aster thermal bands and thermal camera in Polish and Ukrainian mining regions. International Journal of Coal Science \& Technology, 2020. Available at: https://doi.org/10.1007/s40789020-00375-4 (accessed 26 December 2020).

18. Wang Y., Sheng Y., Qiang G., Yue-yue S., Xiu-jun W., Zhi-jie Z. Infrared thermography monitoring and early warning of the spontaneous combustion of coal gangue pile. The International Archives of the Photogrammetry, Remote Sensing and Spatial Information Sciences, Beijing: XXIst International Society for Photogrammetry and Remote Sensing Congress, 2008 , vol. XXXVII. Part B8, pp. 203-206.

19. Kuenzer C., Zhang J., Tetzlaff van Dijk A., Voigt P., Mehl S. Wagner W.H. Uncontrolled coal fies and their environmental impacts: investigating two arid mining regions in north-central China. Applied Geography, 2007, vol. 27, pp. 42-62.

20. Kozyr D.A., Kostenko V.K. Obosnovanye metodiki teplovogo nerazrushayushchego kontrolya poverkhnosti istochnikov goreniya i samonagrevaniya na porodnykh otvalakh ugolnykh shakht [Substantiation of the methodology for thermal non-destructive testing of the surface of combustion and self-heating sources on waste dumps of coal mines]. Problemy nedropolzovaniya: sbornik

Information about the authors

Sergey P. Vysotsky, Dr. Sc., professor, Donbas National Academy of Civil Engineering and Architecture.

Dmitriy A. Kozyr, Cand Sc., associate professor, Donetsk National Technical University. nauchnykh trudov [Subsoil use problems: collection of scientific papers]. St-Petersburg, St Petersburg University Publ., 2016. P. 2, pp. 104-106.

21. Vysotsky S.P., Kozyr D.A. Monitoring of the thermal state of waste dumps using remote control methods. Bulletin of the Academy of Civil Protection: scientific journal, 2018, vol. 1 (13), pp. 59-69. In Rus.

22. Vysotsky S.P., Kozyr D.A. Control of the ecological state of waste dumps. Bulletin of the Donbass National Academy of Civil Engineering and Architecture, 2018, vol. 2018-3 (131), pp. 12-18. In Rus.

23. Kozyr D.A. Usovershenstvovanie metodov kontrolya temperatury pri obespechenii ekologicheskoy bezopasnosti porodnykh otvalov ugolnykh predpriyatiy [Improving temperature control methods while ensuring the environmental safety of waste dumps of coal enterprises]. Problemy prirodopolzovaniya $i$ ekologicheskaya situatsiya $v$ Evropeyskoy Rossii $i$ na sopredelnykh territoriyakh [Problems of nature management and the ecological situation in European Russia and adjacent territories]. Belgorod, POLITERRA Publ. House, 2017. pp. 339-342.

24. Vysotsky S.P., Kozyr D.A. Study of the processes of generation of toxic gas emissions on waste dumps. Bulletin of the Academy of Civil Protection: scientific journal, 2018, vol. 3 (15), pp. 68-74. In Rus.

25. Zborshchik M.P., Osokin V.V. Gorenie porod ugolnykh mestorozhdeniy $i$ ikh tushenie [Combustion of waste of coal deposits and their suppression]. Donetsk, DonGTU Publ., 2000. $180 \mathrm{p}$.

26. Zakharov E.I., Kachurin N.M., Mohnachuk I.I. Physical-chemical properties of coals and coal massifs. Bulletin of TulSU. Earth sciences, 2012, vol. 2, pp. 58-67. In Rus.

27. Lazarevic D.A. In-situ removal of hydrogen sulphide from landfill gas arising from the interaction between municipal solid waste and sulphide mine environments within bioreactor conditions. Stockholm, Royal Institute of Technology, 2007. 87 p.

Received: 22 September 2021. 